\title{
Agent-based investigation of sexually transmitted infection
}

\author{
Dmytro Chumachenko*1 and Tetyana Chumachenko² \\ 'Informatics Department, National Aerospace University "Kharkiv Aviation Institute”, Kharkiv, Ukraine; ${ }^{2}$ Kharkiv National Medical \\ University, Kharkiv, Ukraine
}

\section{Objective}

To develop agent-based model of sexually transmitted infections spreading by example of Syphilis and its analysis.

\section{Introduction}

Every year nearly 12 million new cases of syphilis in the world are registered. Currently, in many countries of the world the stabilization or even reduction of the incidence of syphilis is marked, but this does not apply to Ukraine. The current stage of development of the syphilis problem in Ukraine is characterized by not only high morbidity, but also the fact that in the overwhelming number of cases, we are talking about the latent forms and atypical manifestations of the disease and resistance to therapy [1].

Preventive and prophylactic measures are important in maintaining the public health. Predicting the dynamics of disease spreading allows developing appropriate countermeasures and ensuring rational use of human and material resources. Qualitative forecast of syphilis spreading is possible to implement by means of mathematical modeling

\section{Methods}

Deterministic analytical models that are most common in epidemiological studies do not take into account the dynamic and stochastic nature of epidemics. Agent-based simulation approach to modeling allows fixing these shortcomings. It allows conveying the social structure of simulated system by the most natural and easy way. Each agent has individual state variables and rules of behavior that allows detailing the model very deeply. Therefore there is no need to describe the complex system of mathematical formulas and probability of the dynamics of the epidemic process is defined parametrically. The NetLogo software has been used for the program realization of the developed model.

\section{Results}

The model of morbidity by syphilis spreading has been developed by the tradition SIR model expansion. Thus, agents can be in following states: S (Susceptible) for health people, $\mathrm{I}_{\mathrm{p}}$ (Infected Primary) for infected people who stay in primary stage and can transmit the infection by direct sexual contact with susceptible person, $\mathrm{I}_{\mathrm{S}}$ (Infected Secondary) for infected people who stay in secondary stage and have also infectious skin lesions, $I_{L}$ (Infected Latent) for infected people who stay in latent stage and change its contagious rate from early latent syphilis to late latent syphilis, $\mathrm{I}_{\mathrm{T}}$ (Infected Tertiary) for infected people who stay in tertiary stage and transmit the infection partially, and R (Recovered) for people who are recovered from the infection. Infecting of agents in the model depends on the number and state of agents and the stage of infected agent's disease. Also, in order to correctly determine the intensity of contacts with other agents different age groups of agents have been highlighted in the model. Screen form of developed agent-based model of syphilis spreading is shown in Figure.

The transmission between agent's states are defined by probabilistic way and depends on features of particular states as well as different factors, such as coupling tendency, condom use, commitment, test frequency etc.

The analysis of experiments under developed model has shown that the most influencing factor in the reduction in the percentage of patients is frequency of checks on the disease and isolation of patients, the second most important factor is constancy of sexual partners, the third is the use of condoms, and finally, the fourth is the number of exchangeable partners.

\section{Conclusions}

The agent-based model of syphilis spreading has been developed. The model allows forecasting the morbidity by infection and analyzing the disease by changing the initial data. All data has been checked by the factual statistics on the syphilis incidence in Kharkiv region (Ukraine) from 1975 to 2015 years. The simulation results allow us determining the direction of prevention of syphilis treatment and the main factors in reducing morbidity. As is evident from the simulation results, social factors take precedence over the health care that gives grounds for advocacy in health policy among the population, especially the youth. Developed model can be configured for other sexually transmitted infections by changing the disease transition rules.

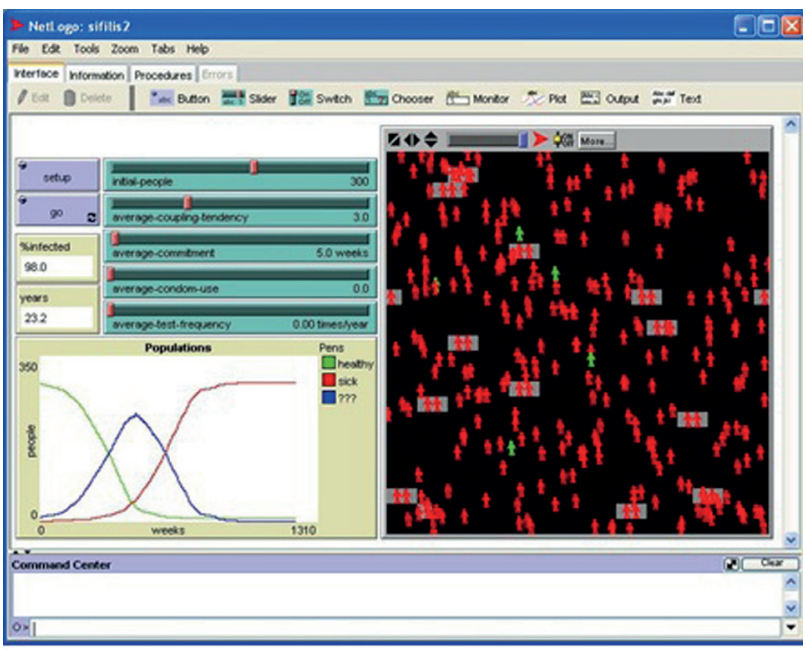

Figure. The main panel of simulation management and graphic visualization.

\section{Keywords}

agent-based simulation; public health; syphilis; mathematical modeling; epidemic process

\section{References}

1. Herbert L. J., Middleton S. I. An estimate of syphilis incidence in Eastern Europe. Journal of Global Health, 2012, 2 (1), pp. 1-7.

\section{*Dmytro Chumachenko}

E-mail: dichumachenko@gmail.com 